\title{
Cognitive-Affective Neural Plasticity following Active-Controlled Mindfulness Intervention
}

\author{
Micah Allen, ${ }^{1}$ Martin Dietz, ${ }^{1}$ Karina S. Blair, ${ }^{2}$ Martijn van Beek, ${ }^{3}$ Geraint Rees, ${ }^{4}$ Peter Vestergaard-Poulsen, ${ }^{1}$ \\ Antoine Lutz, ${ }^{5,6}$ and Andreas Roepstorff ${ }^{1,3}$ \\ ${ }^{1}$ MINDLab, Center of Functionally Integrative Neuroscience, Aarhus University, 8000 Aarhus, Denmark, and ${ }^{2}$ Mood and Anxiety Disorders Program, \\ National Institute of Mental Health, National Institutes of Health, Bethesda, Maryland 20892-2670, ${ }^{3}$ Interacting Minds Centre, Aarhus University, 8000 \\ Aarhus, Denmark, ${ }^{4}$ Institute of Cognitive Neuroscience, University College London, London WC1N 3AR, United Kingdom, ${ }^{5}$ Waisman Laboratory for Brain \\ Imaging and Behavior, University of Wisconsin-Madison, Madison, Wisconsin 53705-2280, and ' $2 y 0 n$ Neuroscience Research Center, Institut National de \\ la Santé et de la Recherche Médicale U1028, CNRS UMR5292, Lyon 1 University, Lyon, France
}

Mindfulness meditation is a set of attention-based, regulatory, and self-inquiry training regimes. Although the impact of mindfulness training (MT) on self-regulation is well established, the neural mechanisms supporting such plasticity are poorly understood. MT is thought to act through interoceptive salience and attentional control mechanisms, but until now conflicting evidence from behavioral and neural measures renders difficult distinguishing their respective roles. To resolve this question we conducted a fully randomized 6 week longitudinal trial of MT, explicitly controlling for cognitive and treatment effects with an active-control group. We measured behavioral metacognition and whole-brain blood oxygenation level-dependent (BOLD) signals using functional MRI during an affective Stroop task before and after intervention in healthy human subjects. Although both groups improved significantly on a responseinhibition task, only the MT group showed reduced affective Stroop conflict. Moreover, the MT group displayed greater dorsolateral prefrontal cortex responses during executive processing, consistent with increased recruitment of top-down mechanisms to resolve conflict. In contrast, we did not observe overall group-by-time interactions on negative affect-related reaction times or BOLD responses. However, only participants with the greatest amount of MT practice showed improvements in response inhibition and increased recruitment of dorsal anterior cingulate cortex, medial prefrontal cortex, and right anterior insula during negative valence processing. Our findings highlight the importance of active control in MT research, indicate unique neural mechanisms for progressive stages of mindfulness training, and suggest that optimal application of MT may differ depending on context, contrary to a one-size-fits-all approach.

\section{Introduction}

Research on mindfulness has expanded dramatically with the rising popularity of mindfulness training (MT) programs. Mindfulness practice consists primarily of focused-attention and open-monitoring practices (Lutz et al., 2008a). Focused attention involves repeated attention fixation, for example, on the sensation of breath entering the nostril, whereas open monitoring

Received June 17, 2012; revised Sept. 7, 2012; accepted Sept. 13, 2012.

Author contributions: M.A., M.v.B., P.V.-P., A.L., and A.R. designed research; M.A. performed research; M.A., M.D., and K.S.B. contributed unpublished reagents/analytic tools; M.A., M.D., and A.L. analyzed data; M.A., G.R., A.L., and A.R. wrote the paper.

This work was supported by a grant from ViFAB (Knowledge and Research Center for Alternative Medicine), Ministry of Health, Denmark, project "Mechanisms of mindfulness training," by grants from the National Center for Complementary and Alternative Medicine (P01-AT004952) and the Adam J. Weissman Foundation (A.L.), by the Velux Foundation (Technologies of the Mind; A.R., M.v.B.), and by a grant from the Wellcome Trust (082334; G.R.). The authors acknowledge the support of the MINDlab Investment Capital for University Research fund. We thank Torben Lund, Christian Gaden Jensen, Ryota Kanai, Chris Frith, and Uta Frith for helpful discussions on the design, analysis, and manuscript. We also thank Mette Steenberg, Karsten Skipper, and Hannah Jakobsen for running the reading and meditation courses. We are deeply grateful to our contemplative collaborators, Jes Bertelsen and Michael Stubberup, whose guidance on the content and aims of meditation training informed this research.

The authors declare no competing financial interests.

Correspondence should be addressed to Micah Allen, Centre for Functionally Integrative Neuroscience, Aarhus University Hospital, Nørrebrogade 44, Building 10G, 5th Floor, 8000 Aarhus C, Denmark. E-mail: micah@cfin.dk.

DOI:10.1523/JNEUROSCI.2957-12.2012

Copyright $\odot 2012$ the authors $\quad 0270-6474 / 12 / 3215601-10 \$ 15.00 / 0$ trains nonjudgmental awareness of moment-to-moment experience (Kabat-Zinn, 2003). The efficacy of MT for treating psychopathology is well established in randomized trials (Grossman et al., 2004). MT improves attention and working memory in healthy novices (Tang et al., 2007; Jha et al., 2010; MacLean et al., 2010), and reduces interference from emotional distractors on a tone discrimination task (Ortner et al., 2007). However, distinguishing the putative causality of affective and cognitive neural mechanisms is difficult given current methodological limitations, including a lack of active-controlled research (Davidson, 2010).

Existing neural data stems from three primary sources: crosssectional studies comparing advanced practitioners and controls (Brewer et al., 2011; Pagnoni, 2012), longitudinal studies using waiting lists (Davidson et al., 2003; Farb et al., 2007) and activecontrolled designs following participants through intensive retreat (Slagter et al., 2007). Collectively these studies associate MT with increased neural activity and gray matter volume in frontoinsular, prefrontal, and limbic regions (Hölzel et al., 2011). However, these findings are limited by several factors. By definition, cross-sectional studies cannot demonstrate causality, and waiting lists are confounded by unmatched demand characteristics and effort (Jensen et al., 2011). The high intensity of retreats 
prevents generalization to MT in mindfulness-naive participants, more typical for clinical applications.

Two studies used active control, but one had limited spatial resolution due to the use of EEG/SPECT (Tang et al., 2009) and the other studied a different type of meditation focused on compassion (Klimecki et al., 2012). Neither study examined executive and affective neural processing simultaneously. MT follows a nonlinear dosage-response curve; early skill training may not reflect later, more advanced practices (Brefczynski-Lewis et al., 2007). At present, it is unclear whether novice MT practices primarily train executive or affective mechanisms, limiting their effective application. We aimed to overcome these limitations by using a longitudinal randomized controlled fMRI study using an affective Stroop (AS) task, which can dissociate cognitive control and emotional interference (Blair et al., 2007). Given the existing knowledge gap regarding the acquisition of meditation-related skills, we examined correlations between individual differences in practice adherence and improvements on attentional and affective measures.

Following from prior evidence (Moore and Malinowski, 2009), we predicted that MT would reduce Stroop conflict and increase central executive blood oxygenation level-dependent (BOLD) activity during effortful cognition. As MT is related to interoceptive and emotional awareness (Lutz et al., 2008b; Silverstein et al., 2011), we expected increases in frontoinsular responses to negative stimuli. MT is thought to regulate cognition through heightened selfawareness (Varela et al., 1992). Therefore, we hypothesized increases in behavioral error awareness (EA). As focused attention is a characteristic element of early MT, whereas nonjudgmental open awareness is typically a more advanced practice, we predicted that alterations in affective processing would be greater in participants with the highest MT practice adherence.

\section{Materials and Methods}

Participants

Meditation-naive participants were recruited from the university and local community in Aarhus, Denmark, via flyers and online announcements. Recruitment materials advertised that participants would receive a free wellness course. Flyers mentioned that the course could include either meditation or similar treatments designed to increase wellness and improve focus. Exclusion criteria were MR incompatibility (metallic implants, claustrophobia, etc.), prior experience with meditation, history of neurological or psychiatric disease, use of prescription drugs, and use of recreational substances within $48 \mathrm{~h}$ of measurement. Inclusion criteria required that all participants be between 18 and 50 years of age, express interest in receiving a wellness course, and be right-handed. Participants gave informed consent in accordance with the local Danish ethics committee. A total of 61 participants were recruited and scanned before randomization to a 6 week mindfulness meditation $(n=30)$ or activecontrol $(n=31)$ condition.

\section{Training protocol}

Participants were briefed at recruitment that they would receive one of two 6 week courses designed to improve emotional well-being and attentional focus. Participants were instructed that the courses were meditation or group reading (our active control), and that any participants completing the study would be invited to participate in the alternative treatment free of cost afterward. As we were interested in the role of intrinsic motivation and practice adherence when randomizing novices to MT, invitation to the alternative follow-up course was the only incentive to participate. Each class consisted of 6 weekly $2 \mathrm{~h}$ meetings. For the mindfulness condition, participants received guided meditation instruction, group activities designed to promote mindfulness, and a CD with further guided meditations for daily homework.

The MT course introduced a mindfulness-style contemplative practice (Lutz et al., 2007), including an additional "heart practice" aimed at developing fullness of feeling (Risom, 2010). The MT course was com- posed of four progressive modules: focused breath awareness, body scanning, compassion, and a fourth open-monitoring practice designed to integrate the prior elements into a unified practice. Our group has previously published cross-sectional research investigating an advanced form of this practice style (Vestergaard-Poulsen et al., 2009). Our intervention thus implemented focused-attention and open-monitoring elements foundational to a wide variety of meditation and mindfulness instruction, such as vipassana, insight meditation, and mindfulnessbased stress reduction (Lutz et al., 2008a), but with additional emphasis on developing fullness of feeling and empathy. Two instructors, with 150 and $2500 \mathrm{~h}$ teaching experience, respectively, taught the course in tan$\mathrm{dem}$. Both instructors had over $10,000 \mathrm{~h}$ of practice in this style.

To control for demand characteristics and treatment effects, we constructed an active-control condition [shared reading and listening (SRL)] to structurally match each group on intervention elements not specific to mindfulness meditation. In an active-controlled intervention, both treatment and control groups contain elements that are hypothesized to improve a target variable (in this case, attentional control and affective processing) to demonstrate the effectiveness of the treatment of interest and isolate key mechanisms, while controlling for nonspecific treatment effects such as placebo, social support, and demand characteristics (Wampold et al., 1997; Davidson, 2010).

As mindfulness has been hypothesized to train attention and affect through interoceptive nonjudgmental awareness (Lutz et al., 2008a; Hölzel et al., 2011), we deliberately manipulated the control condition to deemphasize these elements, isolating their mechanisms of action in MT. Control participants thus received instruction in a specially adapted form of shared reading and listening, in which they met for $2 \mathrm{~h}$ per week to read along and listen while chapters from Jane Austen's Emma (Austen, 2004) were read aloud to them by a qualified instructor, followed by informal group discussion of the plot.

SRL was here hypothesized to cultivate absorption and thoughtrelated processes into an imaginary narrative, in contrast to the presentcentered and open-monitoring aspect of mindfulness. Experimental effects of interest were further isolated through the facilitator leading discussion sessions that placed emphasis on judging the thoughts and motives of the novel's characters, rather than describing participants' emotional or somatic responses to the story. Reading narratives has been used previously as an active control for meditation (Zeidan et al., 2010) and was chosen here as the sessions involve extended reading and listening comprehension, elaboration of plot themes, and an element of social support.

Although several studies on MT used relaxation training as active control (Tang et al., 2007, 2009), we instead selected SRL, as extended listening comprehension is likely to train attention through a high degree of working memory recruitment (Daneman and Merikle, 1996), isolating the interoceptive aspect of MT practice in attentional improvements. We further selected SRL, over, for example, computerized cognitive training or relaxation, to control for well-established effects of social support in emotional well-being and intervention outcome (Turner, 1981; Abbey et al., 1985; Dunst et al., 1986). Given that MT typically involves explicit group exercise such as sharing emotional experiences between participants, SRL is likely to better match for social-support confounds than relaxation training, as reading groups are fundamentally social in nature; SRL sessions were conducted seated around a table, and following each session the facilitator led the group in a collaborative discussion of the plot.

To ensure equivalent instructor enthusiasm and experience in both groups, the reading group instructor held a $\mathrm{PhD}$ in literature and 20 years of experience with "close reading," a literary technique emphasizing sustained, careful evaluation of a text. The SRL instructor received formal training in leading group "shared reading" sessions at the Reader Organization (http://thereader.org.uk/training/). However, as we were interested in controlling for particular aspects specific to a meditation intervention, the participants did not engage in shared reading as taught by the Reader Organization, but rather a novel form of group reading more similar to close reading techniques specially designed for this study as described above. To control for explicit motivation and demand characteristics, all participants were instructed that their class was expected to 

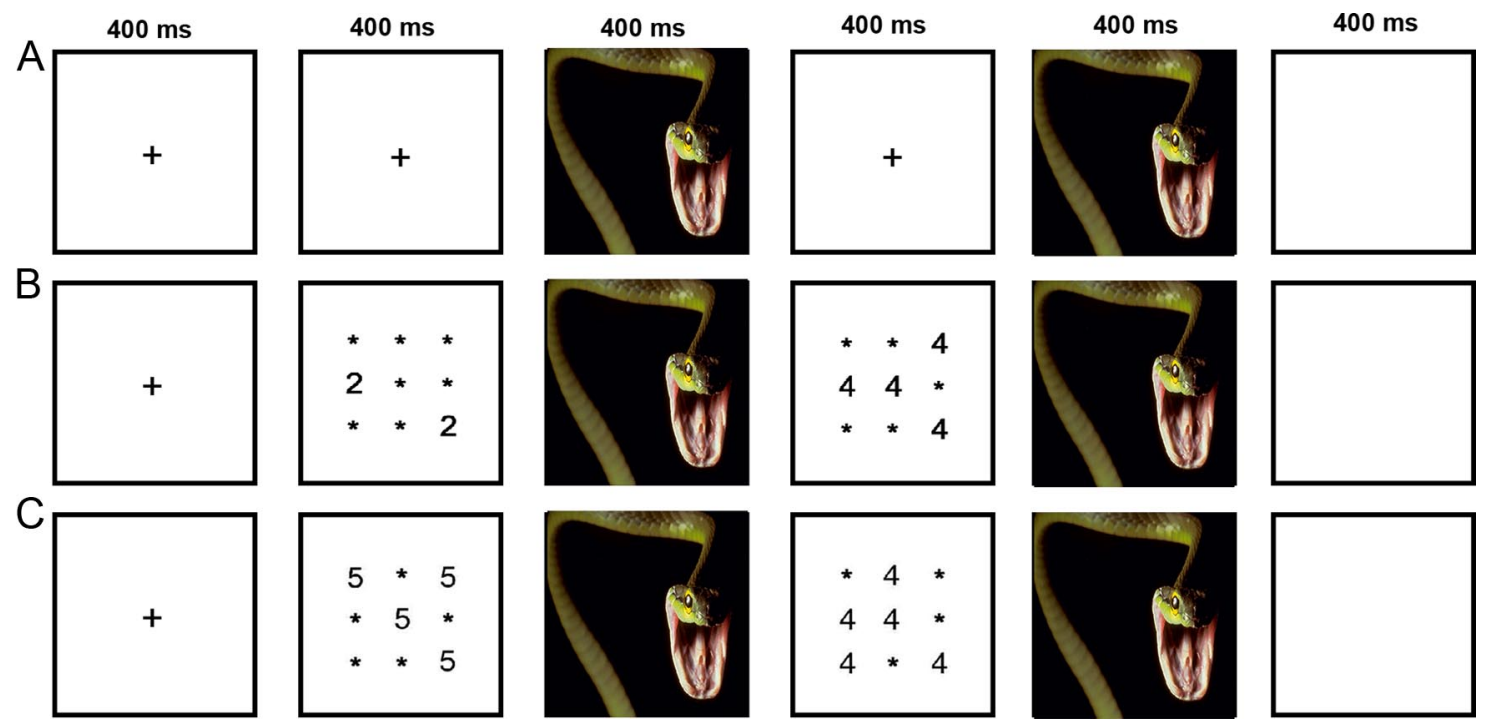

Figure 1. Affective Stroop trial scheme. $\boldsymbol{A}-\boldsymbol{C}$, Each row demonstrates an example of a single stimulus train for that level of task (passive view, $\boldsymbol{A}$; congruent, $\boldsymbol{B}$; and incongruent, $\boldsymbol{C}$ ). IAPS images (third and fifth panels) were randomly selected from 40 negative, neutral, or positive images. Participants were instructed to count the "number of numbers" and to respond as quickly and accurately as possible following the second number display and before onset of the next trial. Response conflict is driven by incongruence between the Arabic numeral and the numeracy of the display (for details, see Materials and Methods).

improve focus and well-being, and that potential benefits would depend on the amount of participation.

Both groups met once per week, in the same building, for $2 \mathrm{~h}$ of instruction every week for 6 weeks. Additionally, participants received a $\mathrm{CD}$ with either 20 min of guided meditation instruction or $20 \mathrm{~min}$ excerpts from Emma. All participants were instructed to complete 20 min of home practice (or listening) per day and to report the minutes practiced and motivation to practice (visual analog scale, $0-100$ ) via an online report. Participants were advised that they should complete as much homework as possible to receive the maximum treatment benefit, but it was emphasized that self-reports should accurately reflect their practice so as to discourage dishonest reporting.

\section{Experimental tasks}

Summary. At each time point (baseline and follow-up measurement), participants came twice to the lab. Time one measurement took place over 2 weeks; participants were notified of their randomized class placement following acquisition of the last time one measurement. The first course took place in the middle of the following week, and thereafter every seventh day for 6 weeks. Follow-up measurement began the week following the last class meeting, and all postmeasures were again obtained over a period of 2 weeks. Participants were instructed to continue their home practice until the follow-up scan. At both time points, participants visited the university hospital for $2 \mathrm{~h}, 1 \mathrm{~d}$ before scanning, for briefing and error-awareness measurements. Additionally, urinary cortisol, heart rate variability, and blood work were obtained on the prescan collection day (data not shown). Scanning occurred on the following day in a $1 \mathrm{~h}$ acquisition session.

Error awareness. To assess treatment-related improvements in metacognitive awareness and executive function, we used the error-awareness task (EAT) (Hester et al., 2005, 2012). Briefly, during the EAT, a stream of color words are presented serially in incongruent font colors. Each word was presented for $900 \mathrm{~ms}$ followed by a $600 \mathrm{~ms}$ interstimulus interval. Participants received training to respond to each word by pressing a left button on "go" trials and to withhold this response during "no-go" trials. There were two no-go conditions; the first was if the same word appeared on two successive trials (repeat no-go), and the second in case of matching word and font-color (color no-go). A commission error was thus any no-go trial where the participant pressed the left button. To report error awareness, participants received training to omit the regular go-trial button response and instead to respond with the alternative (right) button after any commission error. The EAT thus measures the proportion of aware over unaware errors, or the total number of reported errors over the total number of errors (percentage of EA), as well as overall stop accuracy (SA), the total number of correctly withheld no-go trials over the total number of no-go trials. The EAT thus measures both metacognitive awareness (error awareness) and response inhibition (stop accuracy), and recruits a network of regions including salience-related frontoinsula, anterior cingulate, and central executive areas (Ullsperger et al., 2010). All participants completed 45 min of EAT, divided over six runs, each consisting of 250 trials, $1 \mathrm{~d}$ before scanning.

$f M R I$ task. All participants completed eight runs of the AS task, split between premeasurement and postmeasurement sessions (Blair et al., 2007). Briefly, the AS task uses a number counting Stroop task with interleaved images from the International Affective Picture System (IAPS). In the AS, negative IAPS images are associated with additional bottom-up affective processes that increase task difficulty (Blair et al., 2007). In the number-counting Stroop task, congruent trials are those where the Arabic-numeral distractor information is consistent with numerosity (e.g., two $2 \mathrm{~s}$ and four $4 \mathrm{~s}$ ), and incongruent trials are those were the numeral information conflicts with the numerosity (e.g., two 4 s and four 2s) (Fig. 1). IAPS images serve as distractors and interfere with task performance by recruiting additional affective-cognitive resources. The AS utilizes a $3 \times 3$ factorial design, with factors task (incongruent, congruent, and passive view) and emotional valence (negative, neutral, and positive) to assess cognition by emotion interaction.

For this experiment, we chose to restrict our analysis to negative and neutral trials, as positive trials are not thought to interfere with behavior, and we aimed to test specific hypothesis regarding emotional interference (Blair et al., 2007). This is consistent with prior research with the AS in which negative and positive trials are typically analyzed separately (Blair et al., 2007; Vythilingam et al., 2007; Mueller-Pfeiffer et al., 2010). During each number trial, participants viewed a central fixation point, a first numerical display, a picture display, a second number display, and a second picture display of $400 \mathrm{~ms}$ each, ending in a blank stimulus of 1000 ms (Fig. 1). Participants were instructed to determine which numerical display contained greater numerosity and to respond using the right index (first display) or middle finger (second display) as quickly and accurately as possible following the second image display. Before beginning the task, participants were instructed to pay attention to the presented images, as they might be questioned about them later.

Task administration was identical to that of Blair et al. (2007), with all participants completing four sessions at prerandomization and follow- 
up. All stimuli were projected onto a head-coil-mounted mirror using a JVC DLA-HD950 video projector and E-Prime 2.0 software (Psychology Software Tools). Each session consisted of 10 presentations of each emotion by task condition randomized through the session. In addition, 10 fixation trials (presented for the duration of a condition trial, $2500 \mathrm{~ms}$ ) were presented randomly throughout each session, 6 fixation trials were presented in the beginning of each session, and 6 fixation trials were presented at the end of each session. Thus, overall each subject was presented with 40 positive passive view trials, 40 negative passive view trials, 40 neutral passive view trials, 40 positive congruent trials, 40 negative congruent trials, 40 neutral congruent trials, 40 positive incongruent trials, 40 negative incongruent trials, 40 neutral incongruent trials, and 88 fixation trials.

To ensure task compliance, all participants completed one practice session outside the scanner just before entering the MRI. Additionally, participants completed $7 \mathrm{~min}$ of resting state acquisition before task administration, and $\sim 10$ min of a self-awareness task adopted from the study by Farb et al. (2007). These data are not reported in this paper. The order of task administration (self-awareness vs AS task) was counterbalanced across participants. Participants completed $10 \mathrm{~min}$ of high-resolution structural and diffusion tensor imaging following the echoplanar image (EPI) run, not reported here.

fMRI image acquisition and preprocessing. T2*-weighted, gradientecho EPIs were acquired at the Aarhus University Hospital on a Siemens Trio 3T scanner using a 32-channel head coil. Echoplanar images were acquired using an interleaved slice acquisition (TR, $2500 \mathrm{~ms}$; TE, $30 \mathrm{~ms}$; flip angle, $90^{\circ}$; 47 slices of $3 \mathrm{~mm}$ thickness; in-plane resolution, $3 \times 3 \times$ 3; FOV $192 \times 192 \mathrm{~mm}$ ). Soft cushions were used to minimize head movement.

fMRI data analysis and preprocessing was performed using default settings in Statistical Parametric Mapping (SPM8 version 4667; http:// www.fil.ion.ucl.ac.uk/spm) (Friston, 2007). The functional images of each participant were motion corrected and realigned (Friston et al., 1995), spatially normalized to MNI space using the SPM EPI template and trilinear interpolation (Ashburner and Friston, 1999), and smoothed using a $8 \mathrm{~mm}$ full-width at half-maximum smoothing kernel.

Behavioral analysis. All data for the EAT (percentage correct EA and percentage correct SA) were entered into separate two-way repeatedmeasures ANOVAs to assess group by time interactions. For the AS task, mean reaction times (RTs) were calculated for each correct trial and submitted to four-way repeated-measures ANOVA (group by time by task by valence). RTs within each condition were normally distributed (Shapiro-Wilk test of normality, $p>0.1$ ), and no participant performed at $<60 \%$ accuracy (mean task accuracy, Time 1, 91.76\%; Time 2, $96.08 \%)$. As such, the total AS dataset at follow-up was analyzed $(N=$ 38). All ANOVAs were conducted in SPSS 20 (IBM).

For post hoc regression analysis on the impact of individual differences in self-reported amount of practice on behavior, we calculated mean Time 2 minus Time 1 AS conflict RTs (incongruent trials minus congruent trials). We then estimated separate general linear models for the relationship of change over time (Time 2 minus Time 1) in conflict RTs, EAT stop accuracy, and error awareness with amount of practice as a between-group predictor variable. Thus, we conducted $F$ tests to examine differences in between group slopes (i.e., MT slope is not equal to SRL slope) for three independent behavioral measures followed by a post hoc within-group least-squares linear regression. All regression analysis was conducted in MATLAB R2011a (Mathworks).

fMRI analysis - single-subject level. fMRI data were analyzed using a two-level hierarchical general linear model (Friston et al., 1994; 1999). The first-level design matrix modeled fixed effects over the eight runs (four premeasurement and four postmeasurement). Each run was modeled using nine event-related regressors, one for each of the AS conditions (positive, negative, and neutral passive view; positive, negative, and neutral congruent; and positive, negative, and neutral incongruent). In addition, one regressor modeled error/missed trials, and six regressors of no interest contained the realignment parameters to correct for motion artifacts.

To assess task-related neural plasticity, the time by condition interaction was tested at the first level using four linear $t$ contrasts for the positive interaction of time (post $>$ pre) with the main effect of task (incongruent + congruent $>$ passive view), response conflict (incongruent $>$ congruent), emotion (negative $>$ neutral), and the task by emotion interaction. First-level contrasts thus tested for greater BOLD signal at Time 2 for $X$ versus $Y$ (e.g., negative vs neutral) compared to $X$ versus $Y$ at time 1 . We selected the above contrasts as we were primarily interested in investigating the effect of MT on executive function, emotional processing, and their interaction.

fMRI analysis - group level. To assess differences between meditators and controls at the group level, random-effects (RFX) analyses were performed using two-sample $t$ tests for each of the four first-level contrasts of interest: time by task, time by conflict, time by emotion, and time by (task by emotion) (Friston et al., 1999). By passing the time by condition contrast images generated at the first level to the RFX group analysis, we could assess increases and decreases in BOLD signal via MT $>$ SRL and SRL $>$ MT contrasts, further delineated in post hoc testing (see below). Furthermore, to assess the effect of MT practice adherence on BOLD responses, the amount of practice time in minutes was mean centered across participants within each group and modeled as a covariate of interest (i.e., interaction with group means). To assess group differences in BOLD activation related to practice time, $t$ contrasts for total amount of practice in MT > total practice time in SRL were performed for each contrast of interest: time by task, time by conflict, time by emotion, and time by (task by emotion).

All resulting statistical parametric maps were corrected for multiple comparisons using Gaussian random field theory (Worsley et al., 1996) with a cluster-level familywise error (FWE) threshold at $p<0.05$. To determine the directionality of resulting differences, e.g., to differentiate significant increases in MT from decreases in SRL, we conducted within-group post hoc analysis using unidirectional $t$ contrasts (mean MT $>$ baseline or mean SRL $<$ baseline), with FWE cluster-level threshold $p<0.05$.

\section{Results}

\section{Demographics, intervention, and behavior}

At randomization, groups did not significantly differ in mean age (MT, 27; SRL, 26; $\left.F_{(1,60)}=0.59 ; p=0.45\right)$, years of formal education $\left(F_{(1,57)}=1.83 ; p=0.18\right)$, or gender $\left(X_{(1)}^{2}=1.31, N=61\right.$, $p=0.31)$. Twenty-three participants dropped out during the course of the study (10 SRL, $13 \mathrm{MT}$ ). Of those failing to complete the study, 10 cited a lack of time, 5 failed to attend classes, 2 became pregnant, 1 cited an unexpected trip out of the country, and 3 declined to participate in the follow-up measurement. Additionally, two follow-up scans were lost due to technical failure associated with data acquisition and excessive head motion, leaving a total $N$ at study completion of 38 (19 MT, 19 control). At follow-up, groups did not differ in age $\left(F_{(1,37)}<1\right)$, education $\left(F_{(1,37)}<1\right.$ ), or gender (control, 10 female, 9 male; MT, $11 \mathrm{fe-}$ male, 8 male).

An overall motivation score was calculated for each participant by taking the mean of all self-reported motivation-topractice scores. Mean motivation for MT was $60 \%$ [ $\pm 16 \%(\mathrm{SD})]$, SRL 55\% [ $\pm 14 \%$ (SD)]. The MT group practiced, on average, $1066 \mathrm{~min}[ \pm 383 \mathrm{~min}(\mathrm{SD})]$, whereas the control group averaged $1352 \mathrm{~min}[ \pm 286 \mathrm{~min}(\mathrm{SD})]$ SRL. Amount of practice was calculated as the total minutes of practice reported at home plus the total number of minutes spent in class, as recorded by the instructors. Overall, control participants practiced significantly more than MT participants $\left(t_{(37)}=-2.65 ; p=0.01\right)$, but did not differ in their levels of motivation $\left(t_{(37)}=1.02 ; p=0.31\right)$. To explore a possible gender difference in participant's preference for MT or SRL, we conducted a two-way ANOVA with motivation as the dependent variable with factors group and gender. This analysis did not find a significant group by gender interaction on motivation scores $\left(F_{(1,35)}=0.23 ; p>0.5\right)$, indicating that the motiva- 
tion to practice did not differ systematically by gender across the two groups.

Repeated-measures ANOVA did not reveal significant group by time interaction for mean EAT error awareness or stop accuracy $\left(F_{(1,36)}<1\right)$. The main effect of time was significant for EA $\left(F_{(1,36)}=16.35 ; p<0.001\right.$; partial $\left.\eta^{2}=0.31\right)$ and SA $\left(F_{(1,36)}=\right.$ 11.05; $p=0.002$; partial $\left.\eta^{2}=0.24\right)$, indicating that both groups improved significantly on the EAT. The main effect of group was not significant for either measure. At time one, the SRL group exhibited an average of 64 aware errors [ \pm 23 (SD)] and 33 unaware errors $[ \pm 26(\mathrm{SD})]$, whereas the MT group averaged 75 aware errors $[ \pm 24(\mathrm{SD})]$ and 27 unaware errors $[ \pm 17(\mathrm{SD})]$. At time 2, the SRL group exhibited an average of 61 aware errors $[ \pm 25(\mathrm{SD})]$ and 21 unaware errors $[ \pm 18(\mathrm{SD})]$, whereas the MT group averaged 76 aware errors $[ \pm 27(\mathrm{SD})]$ and 17 unaware errors $[ \pm 14(\mathrm{SD})]$. Before regression of EAT scores and practice, error-awareness and stop-accuracy change were examined for outliers, defined as Time 2 minus Time 1 difference scores $>2$ SD from the group mean. One score was removed, driven by poor time one stop performance. Change in EAT stop accuracy was significantly more related to practice hours in MT than in SRL $\left(F_{(1,33)}=5.01 ; p=0.03\right)$. Follow-up within group analysis revealed that amount of MT practice significantly predicted changes in stop accuracy $(r=0.52 ; p=0.028)$ but not SRL $(p>$ 0.1 ) (Fig. 2A). Practice did not significantly predict change in Stroop conflict RTs or EAT error awareness. Thus, while both groups showed improved response inhibition over time, this improvement was only related to practice in the MT group.

For the AS task, we conducted 2 (group, SRL, MT) $\times 2$ (time, pre, post) $\times 2$ (task, congruent, incongruent) $\times 2$ (valence, negative, neutral) repeated-measures ANOVA. As expected, we found significant main effects of task $\left(F_{(1,36)}=111.87 ; p<\right.$ 0.0001 ; partial $\left.\eta^{2}=0.76\right)$ and valence $\left(F_{(1,36)}=7.12 ; p=0.01\right.$; partial $\left.\eta^{2}=0.17\right)$. RTs were slower to incongruent trials indicating Stroop conflict, and slower for negative-valence trials indicating emotional interference. Task by valence was not significant $\left(F_{(1,36)}=1.31 ; p=0.26\right)$, suggesting that conflict during negative Stroop trials was not significantly greater than in neutral Stroop trials. The main effect of time approached significance $\left(F_{(1,36)}=3.52 ; p=0.07\right)$; overall, RTs decreased between sessions. The main effects of group, the time by task interaction, and the group by time by task by valence interaction were not significant $\left(F_{(1,36)}<1\right)$.

Importantly, there was a significant group by time by task interaction for AS RTs $\left(F_{(1,36)}=5.59 ; p=0.02\right.$; partial $\eta^{2}=$ 0.13 ). To determine the nature of this interaction, we conducted post hoc $t$ tests for Time 2 (incongruent minus congruent trials) minus Time 1 (incongruent minus congruent trials) within each group. The $t$ test revealed a significant reduction in Stroop conflict RTs for MT $\left(t_{(18)}=2.42 ; p=0.03\right)$, but not for SRL $\left(t_{(18)}=\right.$ $-0.72 ; p=0.48)$. These results indicate that MT led to a greater reduction in Stroop conflict than in the active-control group (Fig. $2 B$ ), a key finding of the present investigation.

In contrast, the group by time by valence interaction was not significant $\left(F_{(1,36)}<1\right)$; that is, MT did not lead to a treatment effect on valence-related affective RTs. This may have been driven by a significant time by valence interaction $\left(F_{(1,36)}=7.98 ; p=\right.$ 0.01 ; partial $\eta^{2}=0.18$ ); while negative valence slowed RTs at pretest, they did not impact RTs at follow-up. There was not a significant task by group or valence by group interaction; overall task and valence RTs did not systematically vary between groups.
A

MT Practice vs. Stop Accuracy

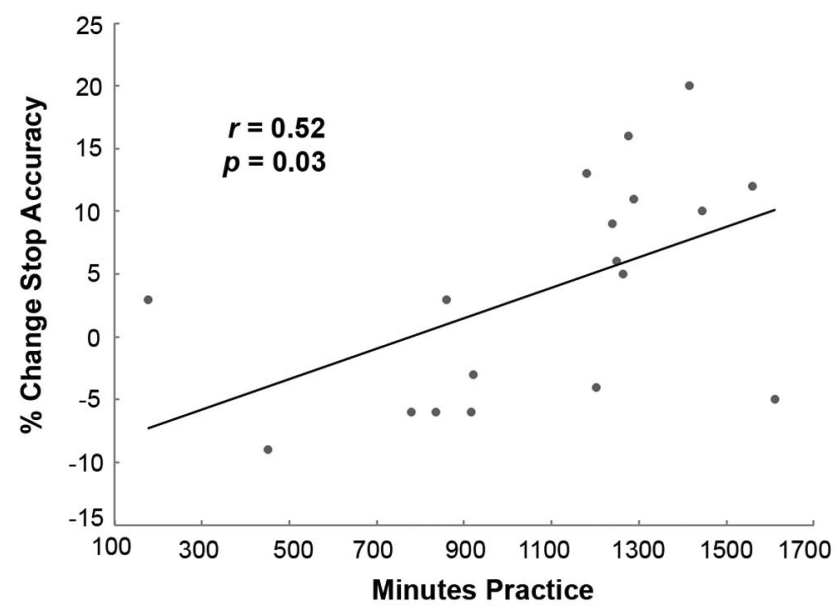

B

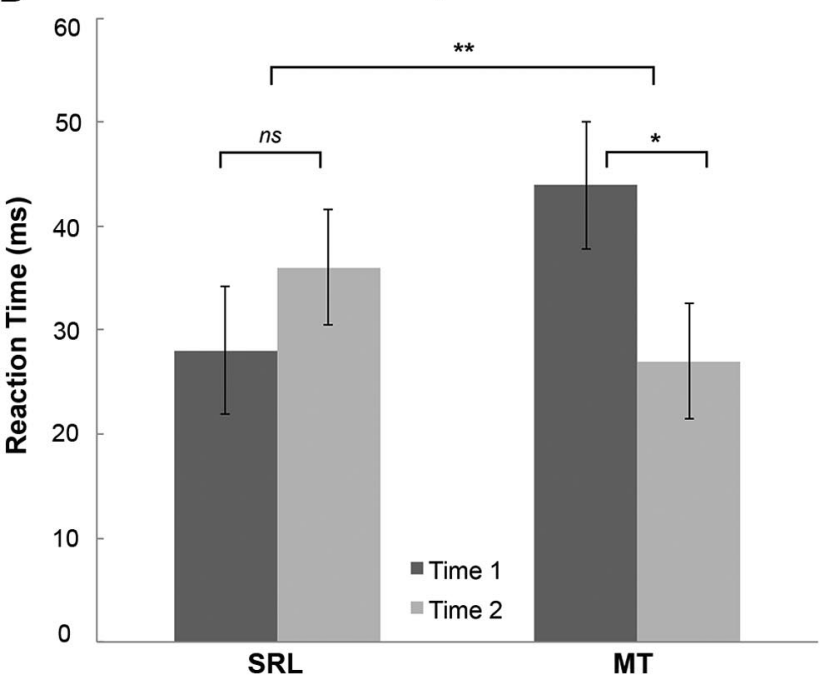

Figure 2. Behavioral results. $A$, Greater MT practice predicts increased EAT stop accuracy. Individual data points are change in percent stop accuracy (mean Time 2 minus mean Time 1) and total practice time for each participant. The MT practice versus $S A$ slope is significantly greater than the SRL versus SA slope $\left(F_{(1,33)}=5.01 ; p=0.032\right.$; data not shown above). Within the MT group (but not SRL) practice significantly predicted $S A$ ( $p=0.03 ; r=0.52$; linear regression line). $\boldsymbol{B}$, Plot of group by time by task interaction. Values represent mean incongruent minus congruent (e.g., Stroop conflict) RT across participants. MT (but not SRL) significantly reduced response conflict. Error bars indicate \pm 2 (SEM). See Results for details. ${ }^{*} p=0.03, t_{(18)}=-2.43$ (within group); ${ }^{* *} p=0.02, F_{(1,36)}=5.59$ (group-by-time interaction). ns, Not significant.

\section{fMRI results}

Mindfulness training effect during task performance

To test the hypothesis that the MT group would demonstrate enhanced BOLD signals after training during task performance, we conducted RFX analysis on the time (post $>$ pre) $\times$ task (congruent + incongruent $>$ passive view) contrast. Group-level contrasts thus indicate significant between group differences in BOLD signal change over time (group by time interactions). For the main effect of task, we detected a significant (FWE clusterlevel threshold, voxel selection, $p=0.001) \mathrm{MT}>\mathrm{SRL}$ effect in left dorsolateral prefrontal cortex (DLPFC) (BA 46; $p=0.03 ; k=$ 223) (Fig. 3A). Post hoc analysis revealed that this result was driven by increased response over time within the MT group in the left DLPFC extending into rostral prefrontal cortex (BA 10; $p=0.04 ; k=203)$. No clusters surviving whole-brain correction were found for the group by time by practice interaction. For the 
effect of response conflict (incongruent $>$ congruent), no voxels survived wholebrain correction in these contrasts.

\section{Mindfulness training effect when} processing negative emotional stimuli To test the hypothesis that MT would increase frontoinsular responses to emotional stimuli, we conducted random effects analysis on the time (pre $<$ post) by emotion (negative $>$ neutral) contrast. For this contrast, no clusters survived whole-brain correction for the MT $>$ SRL contrast. Covariance analysis $(\mathrm{MT}>\mathrm{SRL}$ practice; FWE cluster-level threshold, voxel selection $p=0.001$ ) revealed clusters significantly predicted by increased practice in left posterior insula $(p=0.04 ; k=$ $185)$, left superior frontal gyrus extending into left middle frontal gyrus, anterior cingulate, and frontal superior medial lobule $(p<0.001 ; k=810)$, right frontalorbital gyrus extending into anterior insula $(p=0.002 ; k=350)$, and right frontal superior medial lobule, $p=0.001$, $k=373$.

Post hoc analysis within the MT group for the practice hours contrast revealed significant (FWE cluster-level threshold, voxel selection $p=0.001)$ main effects of time in right anterior insula $(p=0.029$; $k=197)$, left superior frontal gyrus $(p=$ $0.004 ; k=299)$, ACC $(p=0.022 ; k=$ 211), dorsal cingulate $(p=0.009$; $k=260)$, and bilateral frontal superior medial lobule $(p=0.010 ; k=254)$. No voxels survived whole-brain correction in the post hoc SRL practice amount $<$ baseline contrast. These results indicate that, within the MT group, the amount of practice predicted increased frontoinsular activation during emotion processing (Fig. 4).

\section{Task-by-valence interaction}

No clusters survived whole-brain correction for the group by time or practicecovariate contrasts. On an exploratory basis, we lowered the voxel selection threshold to $p=0.01$ and found a significant MT practice $>$ SRL practice cluster in bilateral midcingulate gyrus, extending into the right premotor area $(p=0.01$ FWE corrected on cluster level; $k=998$ ) (Fig. 3B). Post hoc analysis at $p=0.01$ voxel selection threshold revealed this difference to be driven by a positive correlation of practice and right midcingulate/premotor area (FWE cluster level, $p=0.003$ ). See Table 1 for a summary of all fMRI group-level results.

\section{Discussion}

Over the last decade, scientific and public interest in meditation has grown dramatically. Initially, methodological limitations were overlooked in the absence of exploratory pilot data. Here,
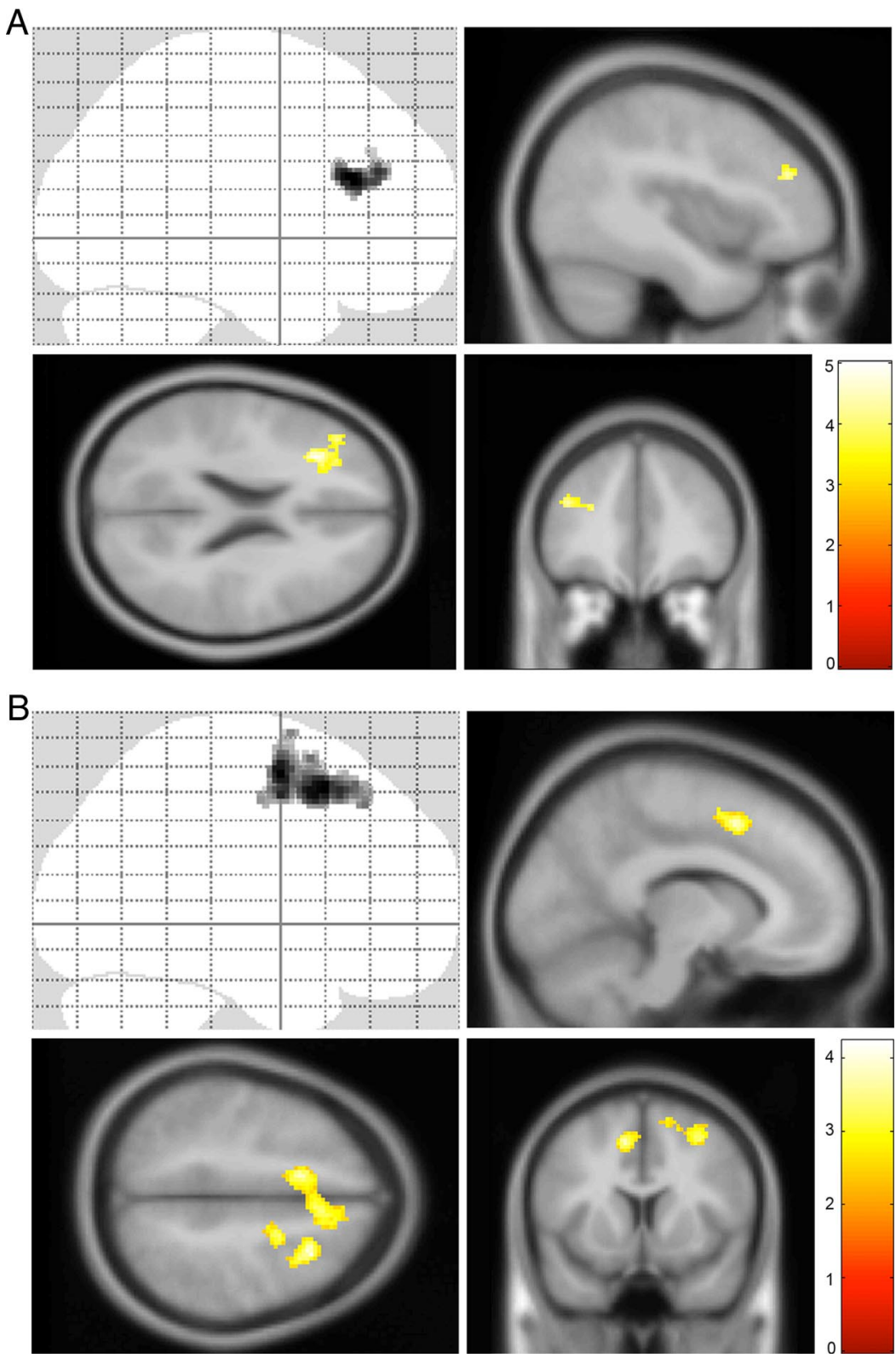

Figure 3. fMRI results. $A$, MT leads to greater left dorsolateral prefrontal cortex responses during task processing than in SRL. Post hoc analysis further revealed this effect to be driven by increases in the MT group. Top left, Sagittal image from SPM "glass brain," which demonstrates spatial extent of activations throughout all slices of the brain simultaneously. The color bar indicates the $t$ statistic associated with each voxel. A whole-brain statistical parametric map (in yellow) is displayed superimposed on sagittal, axial, and coronal views of the standard SPM T1-weighted template, for group by time interaction on the task (incongruent + congruent) $>$ passive view contrast. $p_{\mathrm{FWE}}=0.03$ corrected on cluster level. The voxel selection threshold is $p=0.001$. B , Positive correlation of MT practice and midcingulate cortex and premotor area activation during the task-by-emotion contrast. Post hoc analysis revealed this effect driven by positive correlations in the MT group. A whole-brain statistical parametric map (in yellow) for group by time interaction on the task $>$ emotion contrast is displayed superimposed on coronal and sagittal sections of the SPM T1-weighted template. For the exploratory analysis, cluster selection is $p=0.01 ; p_{\mathrm{FWE}}=0.01$.

we addressed previously unresolved methodological problems including motivation, social support, and demand characteristics in a randomized active-controlled fMRI study. We demonstrated that a 6 week active-controlled mindfulness intervention improved cognitive control and executive-related neural processing in novices. Alterations in affective processing appeared only in those with the greatest amount of MT practice.

Our findings stress the importance of active-controlled design in mindfulness. We observed improvements over time in both 


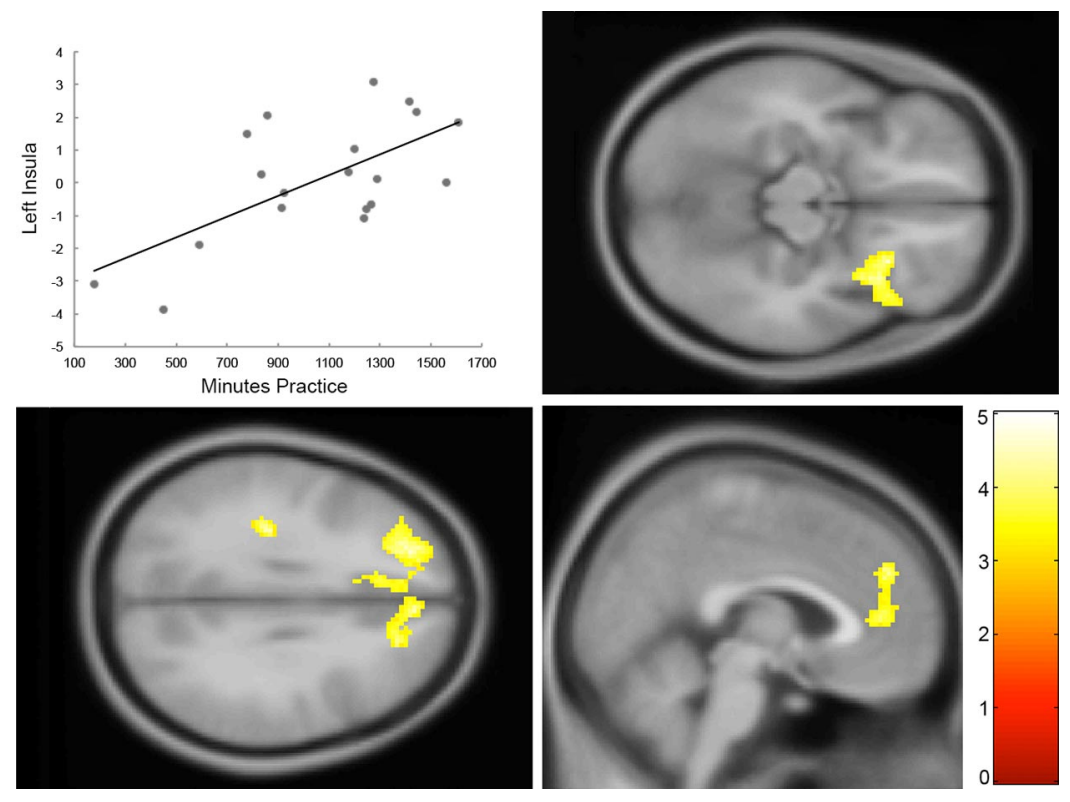

Figure 4. fMRI results. Greater levels of MT practice predict increased dorsolateral prefrontal (bottom left), right anterior insula (top right), and medial-prefrontal BOLD (bottom right) recruitment during negative emotional processing. Post hoc analysis further revealed this effect to be driven by positive correlations in the MT group. Top left, For visualization purposes, BOLD signal was extracted from the peak voxel (left posterior insula) of this contrast and plotted against practice minutes within MT group. The color bar indicates the $t$ statistic associated with each voxel. A whole-brain statistical parametric map (in yellow) is displayed superimposed on coronal, sagittal, and axial views of the SPM T1-weighted template, for group by time interaction on the negative $>$ neutral contrast. $p_{\mathrm{FWE}}<0.05$ corrected on cluster level. The voxel selection threshold is $p=0.001$.

ence strongly predicted recruitment of frontoinsular, cingulate, and midline regions when processing negative stimuli. These regions are involved in bottom-up salience, awareness, and cognitive control (Bush et al., 2000; Seeley et al., 2007; Buckner et al., 2008). In the present study, practice predicted improvements in EAT response inhibition, which has been linked to activation of anterior insula and cingulate cortex (Hester et al., 2005). Here we found positive correlations with BOLD activation in these regions and the amount of mindfulness practice when viewing negative emotional stimuli. We found evidence that attentional modulation, as indexed by the group treatment effects on DLPFC activity and Stroop performance, may be targeted by the kinds of earlier and less intense practices engaged in by the majority of our participants. This accords with phenomenological accounts of MT, where attention stability is a precursor to more advanced open-awareness techniques (Lutz et al., 2008a).

Our findings suggest that affective modulations, indexed here by increased frontoinsular cortex response to negative stimuli and response inhibition, depend

MT and control groups on several measures, including EAT measures (stop accuracy and error awareness) and AS RTs during negative versus neutral trials. Although we did not include a passive-control group, given evidence that similar RT-based measures are easily biased by motivation (Davidson, 2010; Jensen et al., 2011), it is likely that comparison of MT to a waitlist alone would have generated significant treatment effects. Controls completed homework significantly more than MT, expressed equivalent levels of motivation with the training, and actually elected to continue meeting after formal training had ceased. While our design cannot exhaustively rule out differences in participants' perception of the treatment (see below, Limitations), our results seem very unlikely to be due to greater motivation in the MT group.

\section{Differential outcomes of mindfulness training depending on practice adherence}

In the present investigation, MT reduced affective Stroop conflict relative to active control (Fig. $2 B$ ). This is striking in light of the observed DLPFC treatment effect during task performance. DLPFC activity is linked to top-down attention, forming a core node in the central executive network, and supports the online maintenance of information, e.g., working memory (Seeley et al., 2007). DLPFC activation has been associated previously with mindfulness, and MT has protective benefits for working memory (Brefczynski-Lewis et al., 2007; Jha et al., 2010). These results suggest that brief mindfulness training leads to executive neuroplasticity and improved top-down resolution of response conflict, and suggest that attentional improvements reported elsewhere (MacLean et al., 2010; Sahdra et al., 2011) may have a basis in adaptive neuroplasticity of prefrontal central executive networks.

While MT did not impact affective behavior as characterized by mean RT differences, individual differences in practice adher- on practice adherence. This may explain frequently reported MT dosage-response curves in executive regions, in which novices recruit greater central executive activity, whereas advanced contemplatives exhibit reduced activity (Brefczynski-Lewis et al., 2007; Kozasa et al., 2012). One possible interpretation is that early BOLD signal increases reflect an emphasis of focused attention in novices, whereas advanced mindfulness emphasizes affective sensitivity and open awareness.

In an exploratory analysis, greater levels of MT practice predicted increased dorsal ACC and premotor BOLD responses when performing a cognitive task concurrently with negative distractors (i.e., the task by valence interaction), regions linked to affective-interoceptive self-awareness and motor prediction (Seth et al., 2011; Spunt et al., 2012). This finding may support the hypothesis that advanced stages of MT target affective salience and self-awareness.

A important aspect of MT is the goal of transforming skills trained in contemplative states to intrinsic traits, e.g., reacting to experience with intrinsic mindfulness (Hölzel et al., 2011). Previous investigations reported increased medial prefrontal cortex, insula, and anterior cingulate activity during explicit mindfulness practice (Farb et al., 2007; Lutz et al., 2009). We also observed coactivation of these regions during negative emotion trials, implicating increased activation of mindfulnessrelated areas during task performance. As we did not observe the task performance decrements that would likely occur if participants explicitly practiced mindfulness, an intriguing interpretation is that MT participants with the greatest amount of practice responded to emotionally challenging stimuli with intrinsic mindfulness. Collectively our findings suggest that early stages of MT are effective for training cognitive control, with increased affective sensitivity occurring only in our most dedicated participants. 
Table 1. Results for group-level fMRI analysis

\begin{tabular}{|c|c|c|c|c|c|}
\hline Regions & BA & $\mathrm{MNI} x, y, z^{a}$ & $k^{b}$ & Z & $p_{\mathrm{FWE}}{ }^{\mathrm{c}}$ \\
\hline \multicolumn{6}{|l|}{ Task (incongruent $=$ congruent) $>$ passive view } \\
\hline \multirow{3}{*}{ Left middle frontal gyrus } & 46 & $-30,26,22$ & 214 & 4.30 & 0.033 \\
\hline & & $-40,38,24$ & & 3.82 & \\
\hline & & $-28,36,20$ & & 3.71 & \\
\hline \multicolumn{6}{|l|}{ Negative $>$ neutral $^{d}$} \\
\hline \multirow[t]{3}{*}{ Left posterior insula } & 13 & $-32,-24,28$ & 185 & 4.44 & 0.038 \\
\hline & & $-32,-20,40$ & & 3.87 & \\
\hline & & $-38,-14,20$ & & 3.64 & \\
\hline \multirow[t]{3}{*}{ Left middle frontal gyrus, anterior cingulate gyrus } & 10,32 & $-24,44,26$ & 810 & 4.39 & $<0.001$ \\
\hline & & $-32,42,26$ & & 4.18 & \\
\hline & & $-12,30,32$ & & 4.04 & \\
\hline \multirow[t]{3}{*}{ Right lateral frontal- orbital gyrus, right anterior insula } & 47 & $24,24,-22$ & 350 & 4.25 & 0.002 \\
\hline & & $28,22,-14$ & & 4.20 & \\
\hline & & $36,18,-14$ & & 4.03 & \\
\hline \multirow[t]{3}{*}{ Right medial frontal gyrus, right anterior cingulate gyrus } & 9,32 & $6,46,30$ & 373 & 4.04 & 0.001 \\
\hline & & $20,38,30$ & & 3.87 & \\
\hline & & $2,44,12$ & & 3.74 & \\
\hline \multicolumn{6}{|l|}{ Task by emotion } \\
\hline \multirow[t]{3}{*}{ Right middle frontal gyrus, right superior frontal gyrus, anterior cingulate cortex } & 8,32 & $30,16,50$ & 998 & 3.76 & $0.012^{*}$ \\
\hline & & $24,-2,56$ & & 3.60 & \\
\hline & & $-12,12,48$ & & 3.89 & \\
\hline
\end{tabular}

${ }^{a}$ Italics indicate that a peak occurs within the same cluster as the previous peak.

${ }^{b}$ Cluster size in voxels.

'Significant at $p_{\mathrm{FWE}}<0.05$ (cluster-level corrected). The voxel-selection threshold is $p=0.001$.

${ }^{d}$ The group-level contrast corresponds to the practice covariate; MT practice $>$ SRL practice.

*Significant at voxel-selection threshold $p=0.01$, FWE cluster-level corrected $p<0.05$.

\section{Limitations}

We demonstrated that MT leads to neural plasticity during executive task performance. One limitation is the lack of a third passive-control group, which would better dissociate possible roles of motivation and practice in our findings. Here we found equivalent improvement in MT and control groups on response inhibition, whereas only the MT group displayed significantly greater improvement on Stroop conflict scores. One possibility is that the EAT stop-accuracy improvements reflect unique mechanisms for training cognitive control in group listening versus mindfulness, perhaps due to the demanding working memory requirements of listening comprehension (Daneman and Merikle, 1996) compared to the interoceptive nature of MT (Hölzel et al., 2011). Our observation that changes in stop accuracy scores related to practice only in the meditation group is probable evidence for this conclusion. However, it is possible that equivalent practice effects between groups explain these findings.

In addition to equivalent improvements on EAT stop accuracy, we observed that SRL group participants practiced significantly more than MT participants, reported equivalent motivation to practice, and self-selected to continue meeting after cessation of formal training. While these observations strengthen our observed treatment effects, we cannot fully exclude the possibility that the meditation participants may have experienced greater "expectation of improvements" due to an explicit focus during MT on cognitive-emotional performance, leading to an imbalance of task demand characteristics. While these issues might be addressed to some extent by the use of, e.g., relaxation-based controls, our use of SRL groups provides a crucial control for social-support confounds likely to be present in less interactive interventions (for details, see Materials and Methods). These issues are key areas for future research, and could be explored by directly comparing motivation to perform the experimental task, perhaps through online thought sampling (Christoff et al., 2009).
Given that several existing studies use relaxation training as an active control for meditation (Tang et al., 2010), our use of SRL could be seen as a limitation with regard to the generalization of our findings. However, at least one previous behavioral study used narrative reading as a control for meditation (Zeidan et al., 2010). Listening comprehension is linked to working memory demand and as such is an effective control for attention-training elements of mindfulness, isolating the interoceptive mechanisms likely to be at play in an MT intervention. Future work should include a passive and/or relaxation control group in addition to a cognitively demanding active control to better delineate this issue.

\section{Applied mindfulness and neuroplasticity}

A primary finding of the present investigation is our observation of differential improvements depending on individual differences in practice adherence. Here we observed that while a brief ( 6 week) mindfulness intervention provides overall benefits for cognitive control and executive neural processing, alterations at the level of affective processing occurred only in those participants with the greatest level of mindfulness practice. This has possible implications for the understanding and optimal application of MT in clinical settings. Given our finding that alterations in frontoinsular response to emotional stimuli occurred only in those participants with the most practice, application of MT in contexts where training bottom-up affective-somatic processes are a central aim (for example, in sexual affective disorder) may require a more extensive application of $\mathrm{MT}$, perhaps centered on explicit compassion practice (Silverstein et al., 2011). In contrast, treatment effects on DLPFC activity observed here may support the role of brief MT interventions targeting focused attention in, for example, posttraumatic stress disorder, where hypoactivation of DLPFC during the AS task processing has been reported previously (Blair et al., 2012). A one-size-fits-all approach is therefore not likely to lead to the optimal application of MT. 
In summary, this study provides an active-controlled demonstration of executive-related neuroplasticity in a short mindfulness intervention. Our results show that MT is an effective attention-training regime with cascading benefits in cognitive control and affective processing. By controlling for motivation and social support, we found evidence that the constellation of mindfulness practices have particular roles to play in neuroplasticity and mental training. These findings suggest that applications of MT aimed at training attention may benefit from brief intervention, whereas alterations in affective processes require a more substantial commitment of time and motivation.

\section{References}

Abbey A, Abramis DJ, Caplan RD (1985) Effects of different sources of social support and social conflict on emotional well-being. Basic Appl Soc Psychol 6:111-129. CrossRef

Ashburner J, Friston KJ (1999) Nonlinear spatial normalization using basis functions. Hum Brain Mapp 7:254-266. CrossRef Medline

Austen J (2004) Emma. New York: Fine Creative Media.

Blair KS, Smith BW, Mitchell DG, Morton J, Vythilingam M, Pessoa L, Fridberg D, Zametkin A, Sturman D, Nelson EE, Drevets WC, Pine DS, Martin A, Blair RJ (2007) Modulation of emotion by cognition and cognition by emotion. Neuroimage 35:430-440. CrossRef Medline

Blair KS, Vythilingam M, Crowe SL, McCaffrey DE, Ng P, Wu CC, Scaramozza M, Mondillo K, Pine DS, Charney DS, Blair RJ (2012) Cognitive control of attention is differentially affected in trauma-exposed individuals with and without post-traumatic stress disorder. Psychol Med. Advance online publication. doi:10.1017/S0033291712000840. CrossRef Medline

Brefczynski-Lewis JA, Lutz A, Schaefer HS, Levinson DB, Davidson RJ (2007) Neural correlates of attentional expertise in long-term meditation practitioners. Proc Natl Acad Sci U S A 104:11483-11488. CrossRef Medline

Brewer JA, Worhunsky PD, Gray JR, Tang YY, Weber J, Kober H (2011) Meditation experience is associated with differences in default mode network activity and connectivity. Proc Natl Acad Sci U S A 108:2025420259. CrossRef Medline

Buckner RL, Andrews-Hanna JR, Schacter DL (2008) The brain's default network: anatomy, function, and relevance to disease. Ann N Y Acad Sci 1124:1-38. CrossRef Medline

Bush G, Luu P, Posner MI (2000) Cognitive and emotional influences in anterior cingulate cortex. Trends Cogn Sci 4:215-222. CrossRef Medline

Christoff K, Gordon AM, Smallwood J, Smith R, Schooler JW (2009) Experience sampling during fMRI reveals default network and executive system contributions to mind wandering. Proc Natl Acad Sci U S A 106: 8719-8724. CrossRef Medline

Daneman M, Merikle P (1996) Working memory and language comprehension: a meta-analysis. Psychonomic Bull Rev 3:422-433. CrossRef

Davidson RJ (2010) Empirical explorations of mindfulness: conceptual and methodological conundrums. Emotion 10:8-11. CrossRef Medline

Davidson RJ, Kabat-Zinn J, Schumacher J, Rosenkranz M, Muller D, Santorelli SF, Urbanowski F, Harrington A, Bonus K, Sheridan JF (2003) Alterations in brain and immune function produced by mindfulness meditation. Psychosom Med 65:564-570. CrossRef Medline

Dunst CJ, Trivette CM, Cross AH (1986) Mediating influences of social support: Personal, family, and child outcomes. Am J Men Defic 90:403417. Medline

Farb NA, Segal ZV, Mayberg H, Bean J, McKeon D, Fatima Z, Anderson AK (2007) Attending to the present: mindfulness meditation reveals distinct neural modes of self-reference. Soc Cogn Affect Neurosci 2:313-322. CrossRef Medline

Friston KJ (2007) Statistical parametric mapping: the analysis of functional brain images, Ed 1. London: Elsevier/Academic.

Friston KJ, Holmes AP, Worsley KJ, Poline JP, Frith CD, Frackowiak RS (1994) Statistical parametric maps in functional imaging: a general linear approach. Hum Brain Mapp 2:189-210. CrossRef

Friston KJ, Ashburner J, Frith CD, Poline JB, Heather JD, Frackowiak RSJ (1995) Spatial registration and normalization of images. Hum Brain Mapp 3:165-189. CrossRef

Friston KJ, Holmes AP, Price C, Büchel C, Worsley KJ (1999) Multisubject
fMRI studies and conjunction analyses. Neuroimage 10:385-396. CrossRef Medline

Grossman P, Niemann L, Schmidt S, Walach H (2004) Mindfulness-based stress reduction and health benefits: A meta-analysis. J Psychosom Res 57:35-43. CrossRef Medline

Hester R, Foxe JJ, Molholm S, Shpaner M, Garavan H (2005) Neural mechanisms involved in error processing: a comparison of errors made with and without awareness. Neuroimage 27:602-608. CrossRef Medline

Hester R, Nandam LS, O'Connell RG, Wagner J, Strudwick M, Nathan PJ, Mattingley JB, Bellgrove MA (2012) Neurochemical enhancement of conscious error awareness. J Neurosci 32:2619-2627. CrossRef Medline

Hölzel BK, Lazar SW, Gard T, Schuman-Olivier Z, Vago DR, Ott U (2011) How does mindfulness meditation work? Proposing mechanisms of action from a conceptual and neural perspective. Perspect Psychol Sci 6:537-559. CrossRef

Jensen CG, Vangkilde S, Frokjaer V, Hasselbalch SG (2011) Mindfulness training affects attention—or is it attentional effort? J Exp Psychol Gen 141:106-123. Medline

Jha AP, Stanley EA, Kiyonaga A, Wong L, Gelfand L (2010) Examining the protective effects of mindfulness training on working memory capacity and affective experience. Emotion 10:54-64. CrossRef Medline

Kabat-Zinn J (2003) Mindfulness-based interventions in context: past, present, and future. Clin Psychol 10:144-156. CrossRef

Klimecki OM, Leiberg S, Lamm C, Singer T (2012) Functional neural plasticity and associated changes in positive affect after compassion training. Cereb Cortex. Advance online publication. Retrieved June 1, 2012. doi: 10.1093/cercor/bhs142. CrossRef Medline

Kozasa EH, Sato JR, Lacerda SS, Barreiros MA, Radvany J, Russell TA, Sanches LG, Mello LE, Amaro E Jr (2012) Meditation training increases brain efficiency in an attention task. Neuroimage 59:745-749. CrossRef Medline

Lutz A, Dunne JD, Davidson RJ (2007) Meditation and the neuroscience of consciousness: an introduction. In: The Cambridge handbook of consciousness (Zelazo PD, Moscovitch M, Thompson E, eds), pp. 499-551. New York: Cambridge UP.

Lutz A, Slagter HA, Dunne JD, Davidson RJ (2008a) Attention regulation and monitoring in meditation. Trends Cogn Sci 12:163-169. CrossRef Medline

Lutz A, Brefczynski-Lewis J, Johnstone T, Davidson RJ (2008b) Regulation of the neural circuitry of emotion by compassion meditation: effects of meditative expertise. PLoS One 3:e1897. CrossRef Medline

Lutz A, Greischar LL, Perlman DM, Davidson RJ (2009) BOLD signal in insula is differentially related to cardiac function during compassion meditation in experts vs. novices. Neuroimage 47:1038-1046. CrossRef Medline

MacLean KA, Ferrer E, Aichele SR, Bridwell DA, Zanesco AP, Jacobs TL, King BG, Rosenberg EL, Sahdra BK, Shaver PR, Wallace BA, Mangun GR, Saron CD (2010) Intensive meditation training improves perceptual discrimination and sustained attention. Psychol Sci 21:829-839. CrossRef Medline

Moore A, Malinowski P (2009) Meditation, mindfulness and cognitive flexibility. Conscious Cogn 18:176-186. CrossRef Medline

Mueller-Pfeiffer C, Martin-Soelch C, Blair JR, Carnier A, Kaiser N, Rufer M, Schnyder U, Hasler G (2010) Impact of emotion on cognition in trauma survivors: What is the role of posttraumatic stress disorder? J Affect Disord 126:287-292. CrossRef Medline

Ortner C, Kilner S, Zelazo P (2007) Mindfulness meditation and reduced emotional interference on a cognitive task. Motiv Emotion 31:271283. CrossRef

Pagnoni G (2012) Dynamical properties of BOLD activity from the ventral posteromedial cortex associated with meditation and attentional skills. J Neurosci 32:5242-5249. CrossRef Medline

Risom J-E (2010) Presence meditation: the practice of life awareness. Berkeley, CA: North Atlantic.

Sahdra BK, MacLean KA, Ferrer E, Shaver PR, Rosenberg EL, Jacobs TL, Zanesco AP, King BG, Aichele SR, Bridwell DA, Mangun GR, Lavy S, Wallace BA, Saron CD (2011) Enhanced response inhibition during intensive meditation training predicts improvements in self-reported adaptive socioemotional functioning. Emotion 11:299-312. CrossRef Medline Seeley WW, Menon V, Schatzberg AF, Keller J, Glover GH, Kenna H, Reiss AL, Greicius MD (2007) Dissociable intrinsic connectivity networks for 
salience processing and executive control. J Neurosci 27:2349-2356. CrossRef Medline

Seth AK, Suzuki K, Critchley HD (2011) An interoceptive predictive coding model of conscious presence. Front Psychol 2:395. Medline

Silverstein RG, Brown AC, Roth HD, Britton WB (2011) Effects of mindfulness training on body awareness to sexual stimuli: implications for female sexual dysfunction. Psychosom Med 73:817-825. CrossRef Medline

Slagter HA, Lutz A, Greischar LL, Francis AD, Nieuwenhuis S, Davis JM, Davidson RJ (2007) Mental training affects distribution of limited brain resources. PLoS Biol 5:e138. CrossRef Medline

Spunt RP, Lieberman MD, Cohen JR, Eisenberger NI (2012) The phenomenology of error processing: the dorsal ACC response to stopsignal errors tracks reports of negative affect. J Cogn Neurosci 24: 1753-1765. CrossRef Medline

Tang YY, Ma Y, Wang J, Fan Y, Feng S, Lu Q, Yu Q, Sui D, Rothbart MK, Fan M, Posner MI (2007) Short-term meditation training improves attention and self-regulation. Proc Natl Acad Sci U S A 104:17152-17156. CrossRef Medline

Tang YY, Ma Y, Fan Y, Feng H, Wang J, Feng S, Lu Q, Hu B, Lin Y, Li J, Zhang Y, Wang Y, Zhou L, Fan M (2009) Central and autonomic nervous system interaction is altered by short-term meditation. Proc Natl Acad Sci U S A 106:8865-8870. CrossRef Medline

Tang YY, Lu Q, Geng X, Stein EA, Yang Y, Posner MI (2010) Short-term meditation induces white matter changes in the anterior cingulate. Proc Natl Acad Sci U S A 107:15649-15652. CrossRef Medline
Turner RJ (1981) Social support as a contingency in psychological wellbeing. J Health Soc Behav 22:357-367. CrossRef

Ullsperger M, Harsay HA, Wessel JR, Ridderinkhof KR (2010) Conscious perception of errors and its relation to the anterior insula. Brain Struct Funct 214:629-643. CrossRef Medline

Varela FJ, Thompson E, Rosch E (1992) The embodied mind: Cognitive science and human experience. Cambridge, MA: MIT.

Vestergaard-Poulsen P, van Beek M, Skewes J, Bjarkam CR, Stubberup M, Bertelsen J, Roepstorff A (2009) Long-term meditation is associated with increased gray matter density in the brain stem. Neuroreport 20:170-174. CrossRef Medline

Vythilingam M, Blair KS, McCaffrey D, Scaramozza M, Jones M, Nakic M, Mondillo K, Hadd K, Bonne O, Mitchell DG, Pine DS, Charney DS, Blair RJ (2007) Biased emotional attention in post-traumatic stress disorder: a help as well as a hindrance? Psychol Med 37:1445-1455. CrossRef Medline

Wampold BE, Mondin GW, Moody M, Stich F, Benson K, Ahn H (1997) A meta-analysis of outcome studies comparing bona fide psychotherapies: Empiricially, "all must have prizes." Psychol Bull 122:203. CrossRef

Worsley KJ, Marrett S, Neelin P, Vandal AC, Friston KJ, Evans AC (1996) A unified statistical approach for determining significant signals in images of cerebral activation. Hum Brain Mapp 4:58-73. CrossRef Medline

Zeidan F, Johnson SK, Diamond BJ, David Z, Goolkasian P (2010) Mindfulness meditation improves cognition: Evidence of brief mental training. Consciousness and Cognition 19:597-605. CrossRef Medline 